\title{
What's new in management and clearing of airway secretions in ICU patients? It is time to focus on cough augmentation
}

\author{
Nicolas Terzi ${ }^{1,2,3}$ (D), Claude Guerin ${ }^{1,3,4,5,6^{*}}$ and Miguel R. Gonçalves ${ }^{7,8,9}$
}

๑ 2018 Springer-Verlag GmbH Germany, part of Springer Nature

\section{Introduction}

An effective clearing of the airways requires a balance between production and removal of respiratory secretions through the muco-ciliary escalator. In ICU patients, both sides of the balance can be impaired. The amount of respiratory secretions can be increased from infection, aspiration, or swallowing disorders. The removal of respiratory secretions can be reduced by upper airway dysfunction and/or respiratory muscle weakness. Many pre-existing or ICU-acquired situations, like chronic neuromuscular disease (NMD), ICU-acquired neuromuscular weakness, sepsis, delirium or conscious level decline due to brain damage may compromise optimal glottis functioning or respiratory muscle strength [1] and, hence, promote unbalance between production and removal of respiratory secretions. Important pathophysiological consequences of this unbalance include increased airway resistance and work of breathing, hypoxemia, atelectasis, and superinfection. Any caregiver in the ICU, whether he/she is a physician, physiotherapist, respiratory therapist, or nurse, has been used to facing the need of tracheal intubation and invasive mechanical ventilation (after a failing attempt of noninvasive ventilation or high oxygen flow) or extubation failure due to copious respiratory secretions.

The amount of respiratory secretions is a primary cause of extubation failure in the ICU [2] and is likely to delay extubation. Both extubation failure [2] and prolonged invasive mechanical ventilation [3] increase the

*Correspondence: claude.guerin@chu-lyon.fi

1 Service de Médecine Intensive-Réanimation, Centre Hospitalier Universitaire Grenoble, Grenoble, France

Full author information is available at the end of the article risk of poor patient outcome. The need of instrumental techniques and close monitoring from respiratory physiotherapists to manage secretions in specific ICU patients is a reason that prolongs ICU stay with the subsequent increasing costs and hazards. It follows from the above that clearing airway secretions is of utmost importance in the ICU. The optimization of the management of clearing airways in the ICU includes detection of a weak cough and carrying out methods that aim at enhancing the efficacy of coughing.

\section{Cough strength assessment and augmentation}

For the intensivists facing a weaning failure, making the difference between extubation failure from airway issues and weaning failure from mechanical ventilation support need is important and risk factors for each mechanism have been recently investigated [4]. Identifying patients at high risk of post-extubation failure requiring respiratory or cough assistance remains challenging. Several factors may contribute to extubation failure, including cough impairment and presence of thick and/or excessive mucus, in addition to hypoventilation. [5].

Cough assistance and non-invasive mechanical ventilation (NIV) can prevent post-extubation respiratory failure. As these techniques are time-consuming, criteria for selecting those patients most likely to benefit are useful. Previous studies evaluating cough efficiency before extubation focused on peak cough flow (PCF). However, the PCF cut-offs varied widely $[6,7]$, perhaps due to differences in study populations and mechanical ventilation durations. Moreover, diversity of devices used to measure PCF, presence of a cannula used to bypass the upper airway, and differences in the degree of patient coordination and cooperation during measurements may influence

\section{Springer}


the results [7-9]. Recently Terzi et al. [10] assessed cough performance and other easily collected respiratory parameters obtained before and after extubation, with the goal of determining which parameters and measurement conditions best identified patients who would require mechanical assistance after extubation. They found that pre- and post-extubation PCF weakly correlated. Several hypotheses can be suggested to explain this finding. The lack of glottis closure in intubated patients may limit the pressure generated during coughing and, hence, the generated PCF would be less compared to PCF without the tube. Airflow resistance is higher with than without an endotracheal tube. However, forced vital capacity (FVC) measurements before and after extubation are well correlated [10]. FVC may serve as an objective predictor of post-extubation respiratory failure requiring NIV and/ or mechanical cough assistance and/or re-intubation in heterogeneous populations of medical ICU patients. As such, FVC could be used in combination with easily identified risk factors for assessing patients after asuccessful spontaneous breathing trial (SBT), with the goal to identify those likely to require prophylactic pre- and post-extubation mechanical cough assistance (Fig. 1). Gobert et al. [11] found that PCF can be measured using the built-in ventilator flow meter without disconnecting the patient from the ventilator to estimate cough performance. However, PCF alone performed less than combination of PCF and tidal volume to predict postextubation failure, as demonstrated using FVC.

Airway clearance refers to two separate, but connected, mechanisms: muco-ciliary clearance and cough augmentation. Approaches to prevent airway secretion retention in critically ill patients with mechanical ventilation include pharmacotherapy to reduce mucus production or to liquefy secretions, and chest physiotherapy techniques (CPT). Intratracheal saline or sodium bicarbonate or inhaled $\beta_{2}$-agonists are unproven but frequently used pharmacological interventions. CPT can be very effective in preventing pulmonary complications in adult critically ill patients with broncho-pulmonary secretion accumulation. The principles of manual CPT consist of application of manual or mechanical external forces in the thoracic cage that have direct effect on expiratory flow and mucus mobilization. Conventional CPT for secretion management failed to increase weaning and extubation success in one trial [12], which had some limitations [13], but improved patient outcomes in a prospective cohort study [14].

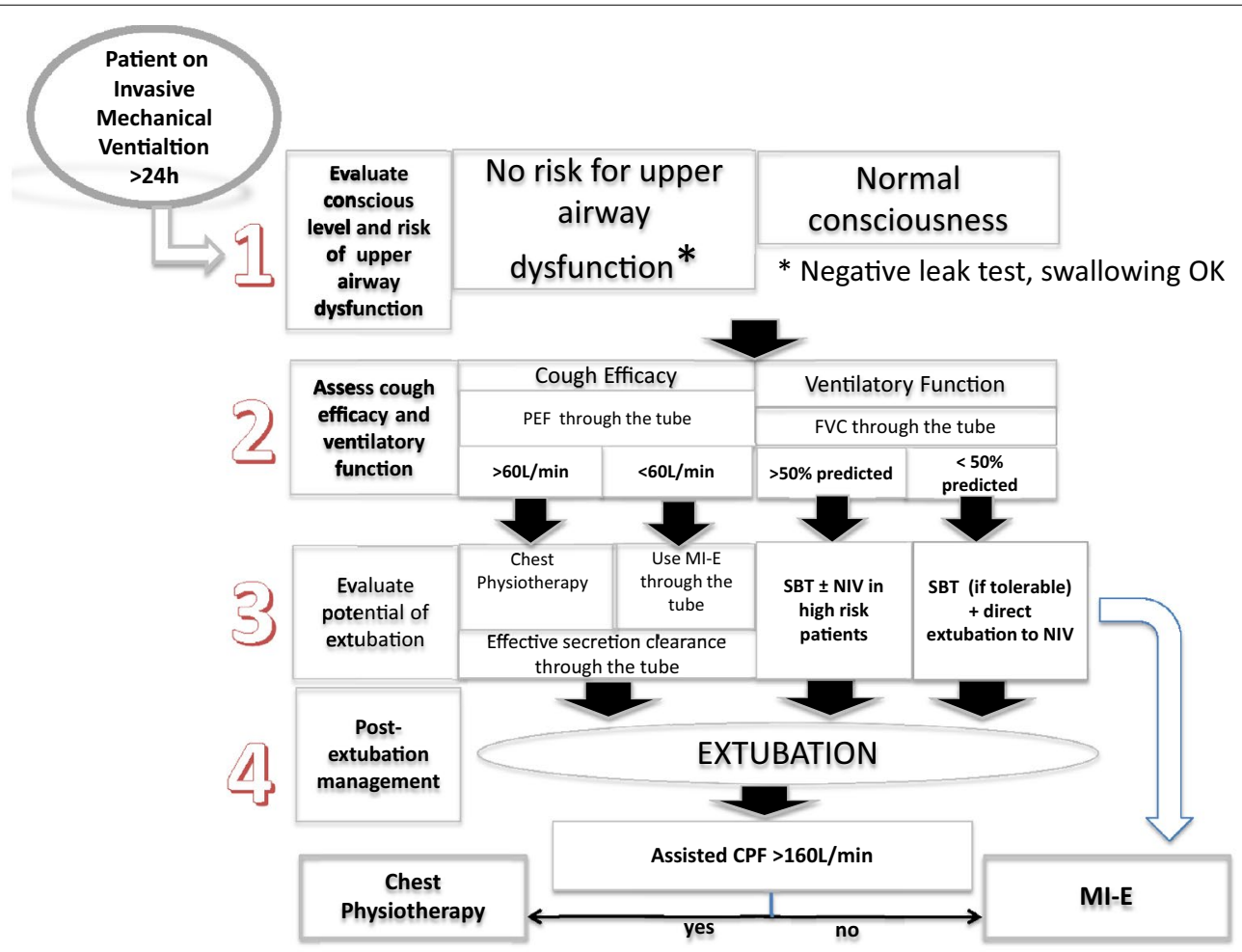

Fig. 1 Decision-making process in an ICU patient under invasive mechanical ventilation focusing on the airway clearance. PEF peak expiratory flow, MI-E mechanical insufflation-exsufflation, CPF cough peak flow, FVC forced vital capacity, SBT spontaneous breathing trial, NIV non-invasive ventilation 
The process of discontinuing mechanical ventilation must balance the risk of complications due to unnecessary delay in extubation with the risk of complications due to early discontinuation and the need for re-intubation. Extubation failure occurs in $10-20 \%$ of patients who meet all weaning criteria and is associated with a higher mortality rate [2]. Therefore, following extubation, all patients should be closely monitored and an early airway secretion clearance must be performed in order to prevent re-intubation.

Mechanical insufflation-exsufflation (MI-E) is a cough assistance therapy in which the device gradually inflates the lungs followed by an immediate change to negative pressure, which produces a rapid exhalation aimed at stimulating the airflow changes that occur during a cough, thereby assisting sputum clearance [15]. MI-E can be provided via an oronasal mask, a simple mouthpiece, or through an endotracheal tube. With the latter, the cuff, when present, should be inflated. The risk of MI-E is baro/volutrauma and hypotension. Intermittent percussive positive ventilation is another means by which to increase secretion clearance.

Although evidence supporting use of MI-E in critically ill patients is lacking [16], Goncalves et al. found that secretion management with MI-E is an useful complementary technique to prevent re-intubation in patients in whom acute respiratory failure develops in the first $48 \mathrm{~h}$ after extubation. In this study, the re-intubation rate related to NIV failure was significantly lower in the MI-E group than in controls [17].

The use of MI-E can avoid intubation in or quickly extubate NMD patients in acute ventilatory failure with no breathing tolerance and profuse airway secretions due to concurrent chest infections. Successful extubation was reported to occur in 155 of 157 unweanable patients with NMD to NIV and MI-E in two centers using a specific goal-directed protocol [18].

Cough strength assessment remains a challenge in ICU patients and its assistance has strong impact on efficient secretion clearance as it may reduce the incidence of post-extubation respiratory failure and contribute to early extubation and, thereby, avert tracheotomy provided that glottic function is sufficient to avoid secretion aspiration. Thus, a different evaluation and treatment paradigm is required for the optimal management of these patients in the ICU.

\footnotetext{
Author details

${ }^{1}$ Service de Médecine Intensive-Réanimation, Centre Hospitalier Universitaire Grenoble, Grenoble, France. ${ }^{2}$ Université de Grenoble, Grenoble, France. ${ }^{3}$ INSERM, U1042, University of Grenoble-Alpes, HP2, 38000 Grenoble, France. ${ }^{4}$ Service de Médecine intensive-Réanimation, Hospices civils de Lyon, Lyon, France. ${ }^{5}$ Université de Lyon, Lyon, France. ${ }^{6}$ INSERM 955, Créteil, France. ${ }^{7}$ Noninvasive Ventilatory Support Unit, Pulmonology Department,
}

São João University Hospital, Porto, Portugal. ${ }^{8}$ Intensive Care Medicine Department, São João University Hospital, Porto, Portugal. ${ }^{9}$ Faculty of Medicine, University of Porto, Porto, Portugal.

\section{Compliance with ethical standards}

Conflicts of interest

The authors declare no conflict of interest.

Received: 15 October 2018 Accepted: 26 November 2018

Published online: 5 December 2018

References

1. Demoule A, Jung B, Prodanovic H, Molinari N, Chanques G et al (2013) Diaphragm dysfunction on admission to the intensive care unit. Prevalence, risk factors, and prognostic impact-a prospective study. Am J Respir Crit Care Med 188:213-219

2. Thille AW, Richard JC, Brochard L (2013) The decision to extubate in the intensive care unit. Am J Respir Crit Care Med 187:1294-1302

3. Beduneau G, Pham T, Schortgen F, Piquilloud L, Zogheib E et al (2017) Epidemiology of weaning outcome according to a new definition. The WIND study. Am J Respir Crit Care Med 195:772-783

4. Jaber S, Quintard H, Cinotti R, Asehnoune K, Arnal JM et al (2018) Risk factors and outcomes for airway failure versus non-airway failure in the intensive care unit: a multicenter observational study of 1514 extubation procedures. Crit Care 22:236

5. Thille AW, Cortes-Puch I, Esteban A (2013) Weaning from the ventilator and extubation in ICU. Curr Opin Crit Care 19:57-64

6. Beuret P, Roux C, Auclair A, Nourdine K, Kaaki M et al (2009) Interest of an objective evaluation of cough during weaning from mechanical ventilation. Intensive Care Med 35:1090-1093

7. Smina M, Salam A, Khamiees M, Gada P, Amoateng-Adjepong Y et al (2003) Cough peak flows and extubation outcomes. Chest 124:262-268

8. Salam A, Tilluckdharry L, Amoateng-Adjepong Y, Manthous CA (2004) Neurologic status, cough, secretions and extubation outcomes. Intensive Care Med 30:1334-1339

9. Duan J, Liu J, Xiao M, Yang X, Wu J et al (2014) Voluntary is better than involuntary cough peak flow for predicting re-intubation after scheduled extubation in cooperative subjects. Respir Care 59:1643-1651

10. Terzi N, Lofaso F, Masson R, Beuret P, Normand H et al (2018) Physiological predictors of respiratory and cough assistance needs after extubation. Ann Intensive Care 8:18

11. Gobert F, Yonis H, Tapponnier R, Fernandez R, Labaune MA et al (2017) Predicting extubation outcome by cough peak flow measured using a built-in ventilator flow meter. Respir Care 62:1505-1519

12. Templeton M, Palazzo MG (2007) Chest physiotherapy prolongs duration of ventilation in the critically ill ventilated for more than 48 hours. Intensive Care Med 33:1938-1945

13. Ntoumenopoulos G (2007) Comment on "Chest physiotherapy prolongs duration of ventilation in the critically ill ventilated for more than 48 hours" by Drs. Templeton and Palazzo. Intensive Care Med 33:2027 (author reply 2028-2029)

14. Castro AA, Calil SR, Freitas SA, Oliveira AB, Porto EF (2013) Chest physiotherapy effectiveness to reduce hospitalization and mechanical ventilation length of stay, pulmonary infection rate and mortality in ICU patients. Respir Med 107:68-74

15. Chatwin M, Toussaint M, Goncalves MR, Sheers N, Mellies U et al (2018) Airway clearance techniques in neuromuscular disorders: a state of the art review. Respir Med 136:98-110

16. Rose L, Adhikari NK, Leasa D, Fergusson DA, McKim D (2017) Cough augmentation techniques for extubation or weaning critically ill patients from mechanical ventilation. Cochrane Database Syst Rev 1:CD011833 
17. Goncalves MR, Honrado T, Winck JC, Paiva JA (2012) Effects of mechanical insufflation-exsufflation in preventing respiratory failure after extubation: a randomized controlled trial. Crit Care 16:R48
18. Bach JR, Goncalves MR, Hamdani I, Winck JC (2010) Extubation of patients with neuromuscular weakness: a new management paradigm. Chest 137:1033-1039 